\title{
SHARP GEOMETRIC MAXIMUM PRINCIPLES FOR SEMI-ELLIPTIC OPERATORS WITH SINGULAR DRIFT
}

\author{
Ryan Alvarado, Dan Brigham, Vladimir MaZ'ya, \\ Marius Mitrea, And Elia Ziadé
}

\begin{abstract}
We discuss a sharp generalization of the Hopf-Oleinik boundary point principle (BPP) for domains satisfying an interior pseudo-ball condition, for non-divergence form, semi-elliptic operators with singular drift. In turn, this result is used to derive a version of the strong maximum principle under optimal pointwise blow-up conditions for the coefficients of the differential operator involved. We also explain how a uniform two-sided pseudo-ball condition may be used to provide a purely geometric characterization of Lyapunov domains, and clarify the role this class of domains plays vis-à-vis to the BPP.
\end{abstract}

The strong maximum principle (SMP) is a cornerstone result in the theory of second-order elliptic partial differential equations, since it permits deriving information about solutions of differential inequalities without any explicit a priori knowledge of the solutions themselves. The traditional formulation of the SMP typically requires the coefficients of the differential operator to be locally bounded (among other things; cf., e.g., $[3,4,13,14])$. Here we present a version of the SMP for second-order, non-uniformly elliptic operators in non-divergence form, in which this assumption is relaxed to an optimal pointwise blow-up condition. We stress that no measurability assumptions are made on the coefficients of the differential operator in question. Specifically, we have the following theorem.

Theorem 1. Let $\Omega$ be a non-empty, connected, open subset of $\mathbb{R}^{n}$, and suppose that a second-order differential operator in non-divergence form (without a zeroth-order term)

$$
L:=-\operatorname{Tr}\left(A \nabla^{2}\right)+\vec{b} \cdot \nabla:=-\sum_{i, j=1}^{n} a^{i j} \partial_{i} \partial_{j}+\sum_{i=1}^{n} b^{i} \partial_{i}
$$

and which is (possibly non-uniformly) elliptic in $\Omega$, has been given. Also, assume that for each point $x_{0} \in \Omega$ and each vector $\xi \in S^{n-1}$ (the unit sphere centered at the origin of $\mathbb{R}^{n}$ ) there exists a real-valued function $\widetilde{\omega}=\widetilde{\omega}_{x_{0}, \xi}$ satisfying

$$
\widetilde{\omega} \in \mathscr{C}^{0}([0,1]), \quad \widetilde{\omega}(t)>0 \text { for each } t \in(0,1], \quad \int_{0}^{1} \frac{\widetilde{\omega}(t)}{t} d t<+\infty,
$$

Received by the editors April 17, 2011.

2000 Mathematics Subject Classification. Primary: 35B09, 35B50, 35J70; Secondary 28A75, 26B05, 49Q10.

Key words and phrases. Boundary Point Principle, Strong Maximum Principle, non-divergence form operator, blow-up, singular drift, semi-elliptic operator, pseudo-ball condition, Lyapunov domain, geometric regularity. 
and with the property that

(3)

$$
\limsup _{\substack{\left(x-x_{0}\right) \cdot \xi>0 \\ x \rightarrow x_{0}}} \frac{(\operatorname{Tr} A(x))+\max \{0, \vec{b}(x) \cdot \xi\}+\left(\sum_{i=1}^{n} \max \left\{0,-b^{i}(x)\right\}\right)\left|x-x_{0}\right|}{\frac{\widetilde{\omega}\left(\left(x-x_{0}\right) \cdot \xi\right)}{\left(x-x_{0}\right) \cdot \xi}((A(x) \xi) \cdot \xi)}<+\infty \text {. }
$$

Let $u \in \mathscr{C}^{2}(\Omega)$ be a function which satisfies the differential inequality $($ Lu $)(x) \geq 0$ for all points $x \in \Omega$. Then if $u$ assumes a global minimum value at some point in $\Omega$, it follows that $u$ is constant in $\Omega$.

This result is quantitatively sharp. To see this, take $\Omega$ to be the $n$-dimensional Euclidean unit ball centered at the origin and consider

$$
L:=-\frac{1}{n+2} \Delta+\vec{b}(x) \cdot \nabla, \quad \text { where } \quad \vec{b}(x):= \begin{cases}|x|^{-2} x & \text { if } x \in B(0,1) \backslash\{0\}, \\ 0 & \text { if } x=0,\end{cases}
$$

and the function $u \in \mathscr{C}^{2}(\overline{B(0,1)})$ given by $u(x):=|x|^{4}$ for each $x \in \overline{B(0,1)}$.

Then

$$
\begin{gathered}
(L u)(x)=0 \text { for each } x \in B(0,1), \\
u \geq 0 \text { in } B(0,1), \quad u(0)=0 \text { and }\left.u\right|_{\partial B(0,1)}=1,
\end{gathered}
$$

which shows that the SMP fails in this case. To understand the nature of this failure, observe that given a function $\widetilde{\omega}:(0,1) \rightarrow(0,+\infty)$ and a vector $\xi \in S^{n-1}$, condition (3) entails

$$
\limsup _{\substack{x \rightarrow 0 \\ x \cdot \xi>0}} \frac{|x|^{-2} x \cdot \xi}{\frac{\widetilde{\omega}(x \cdot \xi)}{x \cdot \xi}}<+\infty
$$

which, when specialized to the case when $x$ approaches 0 along the ray $\{t \xi: t>0\}$, implies the existence of some constant $c \in(0,+\infty)$ such that $\widetilde{\omega}(t) \geq c$ for all small $t>0$. Of course, this would prevent $\widetilde{\omega}$ from satisfying Dini's integrability condition stipulated in (2).

In fact, Theorem 1 is a corollary of a sharp version of the boundary point principle (BPP) stated in Theorem 2 below which, in effect, is the main result of this note. It has been long recognized that the BPP has a distinct geometrical character, both in its formulation and proof. For example, Zaremba [15], Hopf [7] and Oleinik [12] have proved such BPPs in domains satisfying an interior ball condition. Our Theorem 2 provides a sharp version of their results in which the interior ball condition is replaced by an interior pseudo-ball condition, which we will define shortly (cf. (8)). As such, our result is related to the BPPs established in a series of papers beginning in the early 1970s, by Kamynin and Khimchenko (cf. [8-11]), in which they have weakened Zaremba's interior ball condition to a paraboloid condition. This being said, our pseudo-ball involves less demanding assumptions than those imposed on the paraboloid considered by these authors (e.g., we do not require any differentiability conditions on the shape function $\omega$; see (9) and (10) below). Also, significantly, the coefficients of the differential operators for which our theorem holds are not necessarily bounded or measurable (in contrast to $[7,8,10-12]$, and others), the matrix of top 
coefficients is only degenerately elliptic, and the coefficients of the lower-order terms are allowed to blow up at an optimal rate, dictated by the geometry of the domain.

In order to be specific, we need to introduce some relevant notation and terminology. Given

$$
\begin{gathered}
R \in(0,+\infty) \text { and } \omega \in \mathscr{C}^{0}([0, R]) \text { satisfying } \\
\omega(0)=0 \text { and } \omega(t)>0 \text { for all } t \in(0, R],
\end{gathered}
$$

we define the pseudo-ball with shape function $\omega$, apex $x_{0} \in \mathbb{R}^{n}$, amplitude $a>0$, truncation height $b>0$, and direction vector $h \in S^{n-1}$, to be the subset of $\mathbb{R}^{n}$ described as

$$
\mathscr{G}_{a, b}^{\omega}\left(x_{0}, h\right):=\left\{x \in B\left(x_{0}, R\right): a\left|x-x_{0}\right| \omega\left(\left|x-x_{0}\right|\right)<\left(x-x_{0}\right) \cdot h<b\right\} .
$$

It is relevant to note that the family of pseudo-balls makes the transition between truncated circular cones (which occur in the limiting case $\omega \equiv$ const.) and genuine Euclidean balls (or, rather, solid spherical caps, which correspond to the case when $\omega(t) \equiv t)^{1}$

Lastly, a proper, non-empty subset $\Omega$ of $\mathbb{R}^{n}$ is said to satisfy an interior pseudo-ball condition at a point $x_{0} \in \partial \Omega$ with shape function $\omega$ as in (7) if there exist $a, b>0$ and $h \in S^{n-1}$ such that $\mathscr{G}_{a, b}^{\omega}\left(x_{0}, h\right) \subseteq \Omega$.

Theorem 2. Suppose that $\Omega$ is a non-empty, proper, open subset of $\mathbb{R}^{n}$ and that $x_{0} \in \partial \Omega$ is a point with the property that $\Omega$ satisfies an interior pseudo-ball condition at $x_{0}$, say $\mathscr{G}_{a, b}^{\omega}\left(x_{0}, h\right) \subseteq \Omega$, for some parameters $a, b, R \in(0,+\infty)$, direction vector $h=\left(h_{1}, \ldots, h_{n}\right) \in S^{n-1}$, and a shape function $\omega:[0, R] \rightarrow[0,+\infty)$ exhibiting the following features:

$$
\begin{aligned}
& \omega \in \mathscr{C}^{0}([0, R]), \omega(t)>0 \text { for } t \in(0, R], \sup _{0<t \leq R}\left(\frac{\omega(t / 2)}{\omega(t)}\right)<+\infty, \text { and } \\
& \exists C \in(0,+\infty) \text { such that } \frac{\omega\left(t_{1}\right)}{t_{1}} \leq C \frac{\omega\left(t_{0}\right)}{t_{0}} \text { whenever } 0<t_{0} \leq t_{1} \leq R .
\end{aligned}
$$

Also, consider a non-divergence form, second-order, differential operator (without a zeroth-order term)

$$
L:=-\sum_{i, j=1}^{n} a^{i j} \partial_{i} \partial_{j}+\sum_{i=1}^{n} b^{i} \partial_{i},
$$

$$
\begin{gathered}
\text { where } a^{i j}, b^{i}: \Omega \longrightarrow \mathbb{R}, 1 \leq i, j \leq n, \text { are such that } \\
\sum_{i, j=1}^{n} a^{i j}(x) \xi_{i} \xi_{j} \geq 0 \text { for every } x \in \mathscr{G}_{a, b}^{\omega}\left(x_{0}, h\right) \text { and every } \xi=\left(\xi_{1}, \ldots, \xi_{n}\right) \in \mathbb{R}^{n}, \\
\text { and } \sum_{i, j=1}^{n} a^{i j}(x) h_{i} h_{j}>0 \text { for every } x \in \mathscr{G}_{a, b}^{\omega}\left(x_{0}, h\right) .
\end{gathered}
$$

\footnotetext{
${ }^{1}$ The term "pseudo-ball" has been chosen faute de mieux, primarily because of the latter observation.
} 
In addition, suppose that there exists a real-valued function $\widetilde{\omega}$ satisfying

$$
\widetilde{\omega} \in \mathscr{C}^{0}([0, R]), \quad \widetilde{\omega}(t)>0 \text { for each } t \in(0, R], \text { and } \int_{0}^{R} \frac{\widetilde{\omega}(t)}{t} d t<+\infty,
$$

with the property that

$$
\limsup _{\mathscr{G}_{a, b}^{\omega}\left(x_{0}, h\right) \ni x \rightarrow x_{0}} \frac{\frac{\omega\left(\left|x-x_{0}\right|\right)}{\left|x-x_{0}\right|}\left(\sum_{i=1}^{n} a^{i i}(x)\right)}{\frac{\widetilde{\omega}\left(\left(x-x_{0}\right) \cdot h\right)}{\left(x-x_{0}\right) \cdot h}\left(\sum_{i, j=1}^{n} a^{i j}(x) h_{i} h_{j}\right)}<+\infty
$$

and

$$
\limsup _{\mathscr{G}_{a, b}^{\omega}\left(x_{0}, h\right) \ni x \rightarrow x_{0}} \frac{\max \left\{0, \sum_{i=1}^{n} b^{i}(x) h_{i}\right\}+\left(\sum_{i=1}^{n} \max \left\{0,-b^{i}(x)\right\}\right) \omega\left(\left|x-x_{0}\right|\right)}{\frac{\widetilde{\omega}\left(\left(x-x_{0}\right) \cdot h\right)}{\left(x-x_{0}\right) \cdot h}\left(\sum_{i, j=1}^{n} a^{i j}(x) h_{i} h_{j}\right)}<+\infty .
$$

Finally, suppose that $u \in \mathscr{C}^{0}\left(\Omega \cup\left\{x_{0}\right\}\right) \cap \mathscr{C}^{2}(\Omega)$ is a real-valued function satisfying

$$
(L u)(x) \geq 0 \text { for each } x \in \Omega, \quad u(x)>u\left(x_{0}\right) \text { for each } x \in \Omega,
$$

and fix a vector $\vec{\ell} \in S^{n-1}$ satisfying the transversality condition $\vec{\ell} \cdot h>0$.

Then there exist $\varepsilon>0$ for which $x_{0}+t \vec{\ell} \in \Omega$ whenever $t \in(0, \varepsilon)$, a compact subset $K$ of $\Omega$ depending only on the geometrical characteristics of $\mathscr{G}_{a, b}^{\omega}\left(x_{0}, h\right)$, and a constant $\kappa>0$ depending only on the quantities in (15) and (16), $\vec{\ell} \cdot h,\left(\inf _{K} u\right)-u\left(x_{0}\right)$, as well as the pseudo-ball character of $\Omega$ at $x_{0}$, with the property that

$$
\liminf _{t \rightarrow 0^{+}} \frac{u\left(x_{0}+t \vec{\ell}\right)-u\left(x_{0}\right)}{t} \geq \kappa
$$

Clearly, the last condition in (9) is satisfied if $\omega$ is non-decreasing, while (10) is satisfied if $\omega(t) / t$ is non-increasing. Let us also note that no measurability assumptions are made on the coefficients of $L$, and that the class of second-order, non-divergence form, differential operators considered in Theorem 2 is invariant under multiplication by arbitrary positive functions (a desirable feature given that if the BPP holds for an operator $L$ as in (11) and if $\psi>0$ then the BPP continues to hold for $\psi L$ ). In addition, the said class contains all uniformly elliptic, second-order, non-divergence form differential operators with bounded coefficients, granted that the domain $\Omega$ satisfies a pseudo-ball condition at $x_{0} \in \partial \Omega$ whose shape function $\omega$ satisfies Dini's integrability condition (in which scenario (15) and (16) are verified if one takes $\widetilde{\omega}:=\omega$ ).

Most significantly, by means of concrete counterexamples it is possible to show that the BPP described in Theorem 2 is sharp. To see this, consider the case when $\Omega:=\left\{x \in \mathbb{R}_{+}^{n}: x_{n}<1\right\}$, the point $x_{0}$ is the origin in $\mathbb{R}^{n}$, and

$$
L:=-\Delta+\frac{\psi\left(x_{n}\right)}{x_{n}} \frac{\partial}{\partial x_{n}} \quad \text { in } \Omega,
$$

where $\psi:(0,1] \rightarrow(0,+\infty)$ is a continuous function with the property that

$$
\int_{0}^{1} \frac{\psi(t)}{t} d t=+\infty
$$


Then, if

$$
u\left(x_{1}, \ldots, x_{n}\right):=\int_{0}^{x_{n}} \exp \left\{-\int_{\xi}^{1} \frac{\psi(t)}{t} d t\right\} d \xi \quad \text { for each }\left(x_{1}, \ldots, x_{n}\right) \in \Omega,
$$

it follows that $u \in \mathscr{C}^{2}(\Omega)$, the function $u$ may be continuously extended at $0 \in \mathbb{R}^{n}$ by setting $u(0):=0$, and $u>0$ in $\Omega$. Furthermore,

$$
\begin{gathered}
\frac{\partial u}{\partial x_{n}}=\exp \left\{-\int_{x_{n}}^{1} \frac{\psi(t)}{t} d t\right\} \\
\frac{\partial^{2} u}{\partial x_{n}^{2}}=\frac{\psi\left(x_{n}\right)}{x_{n}} \exp \left\{-\int_{x_{n}}^{1} \frac{\psi(t)}{t} d t\right\}=\frac{\psi\left(x_{n}\right)}{x_{n}} \frac{\partial u}{\partial x_{n}} \text { in } \Omega,
\end{gathered}
$$

from which we deduce that $L u=0$ in $\Omega$, and $\lim _{\Omega \ni x \rightarrow 0}(\nabla u)(x)=0$, thanks to $(20)$. Hence, the conclusion of the BPP formulated in Theorem 2 fails in this case (say, for the choice $\left.\vec{\ell}:=(0, \ldots, 0,1) \in \mathbb{R}^{n}\right)$. The sole cause of this breakdown is the inability to find a function $\widetilde{\omega}$ satisfying Dini's integrability condition and such that (16) holds. Indeed, in the setting we are currently considering $\vec{b}(x)=\left(0, \ldots, 0, \psi\left(x_{n}\right) / x_{n}\right)$ for each $x=\left(x_{1}, \ldots, x_{n}\right) \in \Omega$, and since $\Omega$ satisfies an interior pseudo-ball condition at 0 with shape function, say $\omega(t) \equiv t$, and direction vector $h:=(0, \ldots, 0,1) \in S^{n-1}$, condition (16) reduces to (for some fixed $a, b>0$ )

$$
\limsup _{\mathscr{G}_{a, b}^{\omega}(0, h) \ni x \rightarrow 0}\left(\frac{\max \{0, \vec{b}(x) \cdot h\}}{x_{n}^{-1} \widetilde{\omega}\left(x_{n}\right)}\right)=\limsup _{\mathscr{G}_{a, b}^{\omega}(0, h) \ni x \rightarrow 0}\left(\frac{\psi\left(x_{n}\right)}{\widetilde{\omega}\left(x_{n}\right)}\right)<+\infty .
$$

In turn, if true, this would force $\widetilde{\omega}(t) \geq c \psi(t)$ for all $t>0$ small (for some fixed constant $c>0$ ). However, in light of $(20)$, this would prevent $\widetilde{\omega}$ from satisfying Dini's integrability condition. This proves the optimality of condition (16) in Theorem 2. A variant of this counterexample also shows the optimality of condition (15). Specifically, let $\Omega, \vec{\ell}, x_{0}, u$ be as before and, this time, consider

$$
L:=-\left(\sum_{i=1}^{n-1} \frac{\partial^{2}}{\partial x_{i}^{2}}+\frac{x_{n}}{\psi\left(x_{n}\right)} \frac{\partial^{2}}{\partial x_{n}^{2}}\right)+\frac{\partial}{\partial x_{n}} \quad \text { in } \Omega .
$$

Obviously, $L u=0$ in $\Omega$ and, as pointed out before, $\Omega$ satisfies an interior pseudoball condition at the origin with direction vector $h=(0, \ldots, 0,1) \in S^{n-1}$ and shape function $\omega(t) \equiv t$. As such, condition (15) would entail (for this choice of $\omega$, after some simple algebra), $\widetilde{\omega}(t) \geq c \psi(t)$ for all $t>0$ small. In concert with (20) this would, of course, prevent $\widetilde{\omega}$ from satisfying Dini's integrability condition.

Moving on, in the last part of this note we wish to comment on the significance of the pseudo-ball condition appearing in the formulation of Theorem 2, and explain how this may be used for the purpose of describing the analytical regularity (of Euclidean domains) in purely geometrical terms. To set the stage we shall make a few definitions. A proper, non-empty subset $\Omega$ of $\mathbb{R}^{n}$ is said to satisfy a uniform interior pseudo-ball condition near $x_{0} \in \partial \Omega$ provided there exists $r>0$ with the property that $\Omega$ satisfies an interior pseudo-ball condition at each point in $B\left(x_{0}, r\right) \cap \partial \Omega$ with the same shape function, amplitude and truncation height. Going further, a set $\Omega$ as above is said to satisfy a uniform two-sided pseudo-ball condition near $x_{0} \in \partial \Omega$ if both $\Omega$ and $\mathbb{R}^{n} \backslash \Omega$ satisfy a uniform interior pseudo-ball condition near $x_{0}$. Moreover, global versions of 
the last two local conditions are defined naturally, by requiring that the said properties hold near all boundary points.

Next, recall that a proper, non-empty open subset $\Omega$ of $\mathbb{R}^{n}$ is called a domain of class $\mathscr{C}^{1, \omega}$ if, near each of its boundary points, the domain in question may be locally described (up to an isometric change of variables) in terms of upper-graphs of $\mathscr{C}^{1}$ functions whose first-order partial derivatives are continuous with modulus of continuity $\omega$. Of course, this condition amounts to requiring that $\Omega$ is a domain of class $\mathscr{C}^{1}$ with the property that its outward unit normal is continuous with modulus of continuity $\omega$, i.e., $\Omega$ is a Lyapunov ${ }^{2}$ domain (cf. with [6, Chapter I]). Finally, the quality of being of class $\mathscr{C}^{1, \omega}$ near a specific boundary point is introduced analogously. ${ }^{3}$

After this preamble, here is the theorem which provides a purely geometric characterization of the class of locally Lyapunov domains in $\mathbb{R}^{n}$, alluded to (a couple of paragraphs) above.

Theorem 3. Let $\Omega$ be an open, proper, non-empty subset of $\mathbb{R}^{n}$ and suppose that $\omega$ is a function fulfilling the conditions listed in (7), and with the additional property that

$$
\omega \text { is strictly increasing and satisfies } \lim _{\lambda \rightarrow 0^{+}}\left(\sup _{t \in(0, \min \{R, R / \lambda\}]} \frac{\omega(\lambda t)}{\omega(t)}\right)=0 \text {. }
$$

Then, given $x_{0} \in \partial \Omega$, the set $\Omega$ satisfies a uniform two-sided pseudo-ball condition with shape function $\omega$ near $x_{0}$ if and only if $\Omega$ is of class $\mathscr{C}^{1, \omega}$ near $x_{0}$.

As a corollary, if $\partial \Omega$ is compact, then $\Omega$ satisfies a global uniform two-sided pseudoball condition with shape function $\omega$ if and only if $\Omega$ is a domain of class $\mathscr{C}^{1, \omega}$.

As a consequence of Theorem 3, granted that $\omega$ is a function as in (7) and (25), the BPP holds in any domain of class $\mathscr{C}^{1, \omega}$ under the analytical hypotheses specified in Theorem 2 (which include all uniformly elliptic, second-order operators in non-divergence form, with bounded coefficients ${ }^{4}$ ). In particular, the BPP holds for all uniformly elliptic, second-order operators in non-divergence form, with bounded coefficients in domains of class $\mathscr{C}^{1}$ with the property that the modulus of continuity of their outward unit normal satisfies Dini's integrability condition (sometimes called Dini-Lyapunov domains; cf., e.g., [2, pp. 143-144]). In the class of $\mathscr{C}^{1}$ domains, the result just described is geometrically sharp. Indeed, this should be contrasted with the fact that there exists a bounded domain $\Omega$ of class $\mathscr{C}^{1}$, which is even convex and $\mathscr{C}^{\infty}$ near all but one of its boundary points, but for which the BPP fails even for such basic differential operators as $L:=-\Delta$.

To see that this is the case, denote by $\mathbf{0}$ the origin of $\mathbb{R}^{2}$, set $\mathbf{e}_{2}:=(0,1) \in \mathbb{R}^{2}$, and consider the shape function $\omega_{*}(t):=\frac{1}{|\ln t|}$ if $t \in\left[0, \frac{1}{e}\right]$ (with the convention that $\left.\omega_{*}(0):=0\right)$. A careful inspection shows that $\mathscr{G}_{1,1}^{\omega_{*}}\left(\mathbf{0}, \mathbf{e}_{2}\right)$ is of class $\mathscr{C}^{1}$ near $\mathbf{0}$, as well as of class $\mathscr{C}^{\infty}$ near any point in $\partial \mathscr{G}_{1,1}^{\omega_{*}}\left(\mathbf{0}, \mathbf{e}_{2}\right) \backslash\{\mathbf{0}\}$ which is sufficiently close to the origin. In addition, $\mathscr{G}_{1,1}^{\omega_{*}}\left(\mathbf{0}, \mathbf{e}_{2}\right)$ is convex near $\mathbf{0}$. This allows one to construct a bounded, convex, domain $\Omega \subseteq B\left(\mathbf{0}, e^{-1}\right)$ of class $\mathscr{C}^{1}$, such that $\mathbf{0} \in \partial \Omega$, which is $\mathscr{C}^{\infty}$

\footnotetext{
${ }^{2}$ Occasionally also spelled as Liapunov or Lyapunoff.

${ }^{3}$ As is customary, the notation $\mathscr{C}^{1, \alpha}$ is used in lieu of $\mathscr{C}^{1, \omega}$ when $\omega(t) \equiv t^{\alpha}$ for some $\alpha \in(0,1]$.

${ }^{4}$ This already extends G. Giraud's BPP, formulated in [5] for $\mathscr{C}^{1, \alpha}$ domains, $\alpha>0$, and uniformly elliptic operators with bounded coefficients satisfying certain regularity conditions.
} 
near any point in $\partial \Omega \backslash\{\mathbf{0}\}$, and which coincides with $\mathscr{G}_{1,1}^{\omega_{*}}\left(\mathbf{0}, \mathbf{e}_{2}\right)$ near $\mathbf{0}$. Hence, in particular,

$$
\Omega \text { and }\left\{(x, y) \in B\left(\mathbf{0}, e^{-1}\right) \backslash\{\mathbf{0}\}: \sqrt{x^{2}+y^{2}}+y \ln \sqrt{x^{2}+y^{2}}<0\right\}
$$

coincide near $\mathbf{0}$ and, as such, $\Omega$ satisfies an interior pseudo-ball condition at $\mathbf{0} \in \partial \Omega$ with shape function $\omega_{*}$. Next, pick $\varepsilon \in\left(0, \frac{1}{2}\right)$ and define $u: \Omega \cup\{\mathbf{0}\} \rightarrow \mathbb{R}$ by setting $u(\mathbf{0}):=0$ and

$$
u(x, y):=\left(y+\frac{\sqrt{x^{2}+y^{2}}}{\ln \sqrt{x^{2}+y^{2}}}\right)\left(-\ln {\sqrt{x^{2}+y^{2}}}^{-\varepsilon} \quad \text { if }(x, y) \in \Omega .\right.
$$

Then, clearly, $u \in \mathscr{C}^{0}(\Omega \cup\{\mathbf{0}\}) \cap \mathscr{C}^{2}(\Omega)$ and $u(\mathbf{0})<u(x, y)$ for every $(x, y) \in \Omega$. Working in polar coordinates $(r, \theta)$, an elementary calculation (recall that $L:=-\Delta$ ) shows that, in $\Omega$,

$$
(L u)(r, \theta)=\frac{1}{r(-\ln r)^{\varepsilon+3}}\left\{(1-2 \varepsilon \sin \theta)(\ln r)^{2}+(\varepsilon+1)(\varepsilon \sin \theta-2) \ln r+(\varepsilon+1)(\varepsilon+2)\right\} .
$$

Since the squared logarithm in the curly brackets above has a positive coefficient given that $\varepsilon \in\left(0, \frac{1}{2}\right)$, matters may be arranged so that $(L u)(x, y) \geq 0$ at each point $(x, y)$ in $\Omega$. On the other hand, a direct calculation (which uses the fact that $\varepsilon>0$ ) gives that $\lim _{y \rightarrow 0^{+}}\left(\partial_{y} u\right)(x, y)=0$, uniformly in $x$. Thus, ultimately, the lower directional derivative of $u$ at $\mathbf{0}$ along $\mathbf{e}_{2}$ is in fact null and, hence, the conclusion in Theorem 2 fails. The source of this breakdown is the fact that for any continuous function $\omega:[0, R] \rightarrow[0,+\infty)$ and any $a, b>0$ with the property that $\mathscr{G}_{a, b}^{\omega}\left(\mathbf{0}, \mathbf{e}_{2}\right) \subseteq \Omega$, from (26) we deduce that $\omega(t) \geq a^{-1} \mid \ln t^{-1}$ for each $t>0$ sufficiently small. Granted this and given that $\int_{0}^{1 / e} \frac{1}{t|\ln t|} d t=+\infty$, we conclude that $\omega$ necessarily fails to satisfy Dini's integrability condition. In concert with (15) which, in the case when $L=-\Delta$, forces $\widetilde{\omega}(t) \geq c \omega(t)$ for all $t>0$ small, this ultimately shows that $\widetilde{\omega}$ fails to satisfy Dini's integrability condition demanded in (14).

In closing, it is illuminating to point out that when $\omega(t) \equiv t^{\alpha}$, for some fixed $\alpha \in(0,1]$, Theorem 3 provides a purely geometric description of the family of domains of class $\mathscr{C}^{1, \alpha}$. In particular, corresponding to the case when $\alpha:=1$, we have the following purely geometric characterization of domains of class $\mathscr{C}^{1,1}$ : a non-empty, proper, open subset $\Omega$ of $\mathbb{R}^{n}$, with compact boundary, is a domain of class $\mathscr{C}^{1,1}$ if and only if it satisfies a uniform two-sided ball condition. (Recall that the latter condition stands for the demand that there exists $r>0$ such that for every $x \in \partial \Omega$ one may find $h(x) \in S^{n-1}$ for which $B(x+r h(x), r) \subseteq \Omega$ and $B(x-r h(x), r) \subseteq \mathbb{R}^{n} \backslash \Omega$.)

Proofs of the above results, as well as other related matters of interest such as applications to boundary value problems, will appear elsewhere (cf. [1]).

\section{References}

[1] R. Alvarado, D. Brigham, V. Maz'ya, M. Mitrea and E. Ziadé, On the regularity of domains satisfying a uniform hour-glass condition and a sharp version of the Hopf-Oleinik boundary point principle, J. Math. Sci., 176(3) (2011), 281-360.

[2] M. Borsuk and V. Kondratiev, Elliptic boundary value problems of second order in piecewise smooth domains, North-Holland Mathematical Library, Elsevier, 69, 2006. 
[3] L. C. Evans, Partial differential equations, in 'Graduate studies in mathematics', 19, American Mathematical Society, Providence, RI, 1998.

[4] D. Gilbarg and N. Trudinger, Elliptic partial differential equations of second order, SpringerVerlag, Berlin/Heidelberg/New York, 1983.

[5] G. Giraud, Problèmes de valeurs à la frontière relatifs à certaines données discontinues, Bull. Math. France, 61 (1933), 1-54.

[6] N. M. Gunther, Potential theory, Ungar, New York, USA, 1967.

[7] E. Hopf, A remark on linear elliptic differential equations of second order, Proc. Amer. Math. Soc. 3 (1952), 791-793.

[8] L. I. Kamynin and B. N. Himčenko, On theorems of Giraud type for a second order elliptic operator weakly degenerate near the boundary, Dokl. Akad. Nauk. SSSR 224(4) (1975), 752755 (in Russian). English translation in Soviet Math. Dokl. 16(5) (1975), 1287-1291.

[9] L. I. Kamynin and B. N. Khimchenko, The maximum principle for an elliptic-parabolic equation of the second order, Sibirsk. Mat. Zh. 13 (1972), 773-789 (in Russian). English translation in Siberian Math. J. 13 (1972).

[10] L. I. Kamynin and B. N. Khimchenko, Theorems of Giraud type for second order equations with a weakly degenerate non-negative characteristic part, Sibirsk. Mat. Zh. 18(1) (1977), 103-121 (in Russian). English translation in Siberian Math. J. 18 (1977).

[11] L. I. Kamynin and B. N. Khimchenko, Investigations of the maximum principle, Dokl. Akad. Nauk. SSSR 240(4) (1978), 774-777.

[12] O. A. Oleinik, On properties of solutions of certain boundary problems for equations of elliptic type, Mat. Sbornik N.S. 30(72) (1952), 695-702.

[13] P. Pucci and J. Serrin, The maximum principle, in 'Progress in nonlinear differential equations and their applications', 73, Birkhäuser, Basel-Boston-Berlin, 2007.

[14] M. Taylor, Partial differential equations. Basic theory, Springer-Verlag, New York, USA, 1996.

[15] M. S. Zaremba, Sur un problème mixte relatif à l'équation de Laplace, Bull. Intern. Acad. Sci. (Cracovie) Sér. A, Cl. Math. Nat. (1910), 313-344.

Department of Math., University of Missouri at Columbia, Columbia, MO 65211, USA

E-mail address: rjamt9@mail.missouri.edu

E-mail address: dob97b@mail.missouri.edu

E-mail address: mitream@mail.missouri.edu

E-mail address: etzm7f@mail.missouri.edu

Department of Math. Sciences, University of Liverpool, Liverpool L69 3BX, UK, and

Department of Math., Linköping University, Linköping SE-581 83, Sweden

E-mail address: vlmaz@mai.liu.se 